\title{
La evaluación de competencias desde el planeamiento didáctico. Conceptualización e importancia
}

\author{
The evaluation of competencies from the didactic planning. \\ Conceptualization and importance
}

Jarrison Caicedo González

Universidad Metropolitana de Ciencia y Tecnología. C. de Panamá, Panamá

Recibido: 05/08/2021 Revisado: 20/09/2021_Aceptado: 25/11/2021Ｐublicado: 15/01/2022

\section{RESUMEN}

Pensar en una evaluación por competencias desde el planeamiento didáctico, es hoy en día una tendencia que debe permear en el mundo académico, esto para que se pueda desarrollar de manera eficiente el plan de estudio o programa educativo que pretende aplica la evaluación como aquella herramienta de mejoramiento en los aprendizajes. Este ensayo tiene el objetivo de acercar al lector hacia un fundamento teórico y conceptual sobre la evaluación de competencias a partir del planeamiento didáctico. Se realizó un rastreo bibliográfico sobre la evaluación, competencias y planeamiento didáctico para dar rigurosidad a los argumentos expuestos. Se entiende entonces, que la evaluación de competencias desde el planeamiento didáctico es crucial al momento de generar estrategias de desarrollo cognitivo porque ayuda a la comprensión de la complejidad del proceso de enseñanza-aprendizaje.

Palabras claves: Evaluación, competencias, planeamiento didáctico, evaluación de competencias.

\begin{abstract}
Thinking about competency-based assessment from didactic planning is nowadays a trend that should permeate the academic world, in order to efficiently develop the study plan or educational program that intends to apply assessment as a tool for learning improvement. The purpose of this essay is to provide the reader with a theoretical and conceptual foundation on the evaluation of competencies based on didactic planning. A bibliographic search on evaluation, competencies and didactic planning was carried out in order to give rigor to the arguments presented. It is then understood that the assessment of competencies from didactic planning is crucial when generating cognitive development strategies because it helps to understand the complexity of the teaching-learning process.
\end{abstract}

Keywords: Assessment, competencies, didactic planning, competence evaluation. 
La evaluación de competencias desde el planeamiento didáctico.

Conceptualización e importancia

\section{INTRODUCCIÓN}

"El maestro enseña más con lo que es, que con lo que dice" (Soren Kierkegaard). La búsqueda de una educación con calidad hace visible los procesos que deben desarrollarse dentro del sistema educativo para la armonización de los aprendizajes que se ofrecen como logros u objetivos de la enseñanza. Aquí entra la evaluación como aquel proceso que busca garantizar el desarrollo de habilidades, capacidades y actitudes dentro del aula.

Resulta oportuno afirmar que la evaluación se plantea como herramienta de mejoramiento de la calidad educativa por los diversos procesos que con ella se logran armonizar, por eso es conveniente hacer cambios significativos en las metodologías que dirigen el quehacer pedagógico.

El siguiente texto es un ensayo que tiene el propósito de hacer una aproximación teórica y conceptual desde la argumentación del autor sobre la evaluación de competencias desde el planeamiento didáctico, y acercarse a su conceptualización e importancia. Se compone de tres partes básicamente; primeramente, se hace una conceptualización de evaluación, evaluación de competencias, evaluación de aprendizajes y planeamiento didáctico, después se dan argumentos sobre los desafíos actuales que enfrenta la evaluación de competencias desde el planeamiento didáctico, y finalmente, se ofrecen algunas conclusiones relativas al tema en discusión.

\section{DESARROLLO}

\section{Hacia una Aproximación Conceptual de Evaluación y Competencias}

Antes de referirnos al tema que nos ocupa, la evaluación de competencias desde el pla- neamiento didáctico, hay que referirnos al marco conceptual de evaluación, competencia y luego su vínculo con el planeamiento didáctico.

Desde la perspectiva más general, se define la evaluación como aquella actividad humana que es inherente a sus acciones, que requiere ser sistemática y que su fin será hacer la valoración de todo lo visible. Es decir, descubrir el valor de una realidad especifica en la que ocurren procesos que producen valiosa información, útiles para la toma de decisiones posteriores a la valoración inicial. Dentro del proceso educativo, la evaluación actúa para tomar el control sobre el grado de cumplimiento de logros, metas u objetivos predichos en el aprendizaje.

De la orden (como se citó en Morales, 2001) refiere que la evaluación es un proceso donde el recogimiento y análisis de información se utilizan para reconocer las perspectivas de la realidad educativa, con ello se podrá lanzar juicios valorativos acerca de los criterios establecidos para el logro del aprendizaje y tomar consecutivamente decisiones asertivas para su mejoramiento. Por lo tanto, el proceso evaluativo ayudará a determinar en qué medida se han alcanzado los objetivos educativos dentro de la enseñanza (Tyler, 1950).

Es importante que se aplique la evaluación dentro de los procesos educativos porque permite el fortalecimiento y la consolidación del aprendizaje, al igual que los logros, metas u objetivos de cualquier campo del saber. A través de la evaluación se puede hacer un diagnóstico certero de las necesidades, que, desde una visión educativa, se requieren suplir vinculando el saber con el desempeño porque representan el procedimiento pedagógico que conducirá hacia la calidad anhelada 
en todo el sistema educativo. Siendo las cosas así, con el desarrollo de la practica pedagógica los actores del entorno educativo pueden aportar para la creación de un modelo de evaluación que devele los objetivos que se persigan en cuanto al qué y el cómo sin limitarse solo a lo que acontece dentro del proceso enseñanza-aprendizaje, sino expandirse integralmente a partir de experiencias y alternativas pedagógicas. Por esta razón, la evaluación resulta ser "una actividad continua del mismo proceso educativo" (Fernández, 2018).

En la actualidad, se conoce la evaluación como un condensado de evidencias que admiten la recolección de datos valiosos sobre el trabajo de los estudiantes en relación a los aprendizajes alcanzados y los objetivos propuestos. No obstante, hay que resaltar que la valoración que hace el docente sobre el desempeño del estudiante mediante la evaluación debe tener como base una propuesta de mejora para que adquiera el carácter de pertinente y meritoria con la que se le pueden hacer transformaciones significativas al aprendizaje y la enseñanza.

Como se ha dicho que la evaluación hace parte integral del proceso educativo, y por ende del aprendizaje del estudiante guiado por las metodologías didácticas del maestro, considera Lemus (s.f.) que esta a su vez deberá hallar correspondencia con los siguientes principios:

Integralidad. Debe hacerse con la participación de todos los actores de la acción educativa donde cada uno definirá los aspectos a mejorar sobre su desempeño y valoración hecha a partir de la evaluación. Incluirá entonces, el avance del estudiante, el método del docente, la práctica pedagógica, la participación colectiva de la institución y el contexto.
Continuidad. Es una actividad continua, adaptable y eficaz que dentro del proceso educativo ayuda a determinar los conocimientos adquiridos por los estudiantes.

Individualidad. Será ajustable a la personalidad y cualidades de cada evaluado como característica particular de su proceso de aprendizaje.

Cientificidad. Permite el acceso a la información a partir de situaciones didácticas y criterios adaptados al aprendizaje.

Diferencialidad. Debe responder a diversos propósitos donde el estudiante pueda descubrir diversos aprendizajes y aportar evidencias sobre su rendimiento.

Finalidad. Le corresponde un objetivo natural del aprendizaje planteado al evaluado, expondrá las razones de la meta estipulada.

Los anteriores principios permiten identificarel papelintegrador de la evaluación dentro del proceso de enseñanza que lidera el docente al momento de plantearse una metodología para orientar el aprendizaje de sus estudiantes.

\section{Evaluación y Competencias}

Como lo hacen notar Chaviano, Baldomir, Coca y Gutiérrez (2016) la evaluación “conduce a la dirección efectiva del proceso enseñanza aprendizaje y a la determinación de su grado de eficiencia" de manera que permite hacer la reflexión constante de las practicas evaluativas que se realizan en el quehacer educativo para orientar el aprendizaje. Aquí el rol del docente adquiere dinamismo en su práctica para acreditar el conocimiento del estudiante. Podemos hacer visible entonces una tendencia 
de la evaluación donde se percibe como como una actividad comprehensiva que demarca la manera como debe aplicarse y la manera como el estudiante la asume para su aprendizaje.

Los cambios estructurales que se han ido implementando en los procesos formativos de los estudiantes para la obtención de su aprendizaje mediante prácticas evaluativas, ha provocado que estos se representen en competencias que al mismo tiempo han demandado modificaciones a las metodologías y el currículo que dimiten en el diseño de un sistema de evaluación por competencias (Cano, 2008). De acuerdo a esta postura, la evaluación queda huérfana y es despojada de la enseñanza y el aprendizaje, lo cual es inconveniente para seguir en la transformación de las prácticas evaluativas que buscan mejorar el diseño de metodologías didácticas.

El término competencias en los últimos años ha ido adquiriendo gran relevancia en el ámbito social, político, educativo, organizacional, universitario, etc., lo que permite considerarla como un concepto polisémico que atiende a múltiples definiciones por autores y organizaciones que pueden ser resumidas brevemente como "saber hacer en contexto". Es decir, se le puede atribuir como la capacidad que tiene una persona para saber hacer una tarea, algo o desempeñarse en un cargo. Como complemento resulta interesante tener en cuenta que el hacer hará referencia a la actividad de la persona que de manera integral agrega el saber, el ser y el convivir en un contexto determinado para desenvolverse y cumplir unos objetivos propuestos.

Conviene subrayar que la capacidad atribuida a las personas, plantea Graham (1991) permiten el desarrollo de la habilidad que adquiere el individuo para realizar alguna actividad como requerimiento de su responsabilidad partiendo de la organización y planificación de acciones concretas que determinan el desempeño de sus funciones.

Nos basaremos ahora en el ámbito educativo para conceptualizar las competencias, para ello, traemos a colación la definición de Llanio (2010) quien afirma que se puede comprender como la capacidad que dentro de la educación "se relaciona directamente con el saber-hacer por el estudiante o el egresado de una profesión" permitiendo que el conocimiento se pueda convertir en acción (Irigoin y Vargas, 2002). Por lo anterior, no cabe duda de que cualquier estudiante estará en la capacidad de producir el conocimiento, desarrollar competencias y actitudes que le permitan enfrentarse a la vida. Entonces, desde el campo educativo las competencias podrán ser definidas como la oportunidad que se le ofrece al estudiante mediante la practica pedagógica de aprender a conocer, a hacer, a vivir juntos, y a ser (Delors, 1996). Son entonces aprendizajes, destrezas y saberes que desarrollan los alumnos para intervenir o participar en el mundo que habita.

Con el fin de lograr un nivel de calidad deseado desde el sistema educativo, las competencias representan en los estudiantes criterios que debe saber y saber hacer desde las áreas que se les enseña, las cuales deben ser diseñadas con metodologías flexibles y alternativas pedagógicas para que pueda poner a prueba las habilidades metacognitivas en la producción de su conocimiento.

En la opinión de Tobón (2006) las competencias "son un enfoque para la educación y no un modelo pedagógico" porque traen consigo algunos atributos particulares, ta- 
les como; integrar el conocimiento con los procesos cognitivos, capacidades y actitudes para resolver situaciones problemáticas; también, programar la formación del estudiantes con base en las condiciones del contexto donde ocurre la acción educativa; y además, dar ruta a la educación alineada a parámetros o estándares de calidad que se desarrollan en la praxis pedagógica.

De estas circunstancias nace el hecho de que las competencias adquieren un carácter formativo, análogamente con los principios de la evaluación, tales como: generalidad; no reconoce un solo aprendizaje, más bien indaga por conceptos y proceso cognitivos. Integralidad; permiten el desarrollo comprensivo de aprendizajes haciendo una valoración reflexiva de sus capacidades intelectuales. Contextualización; obedece a la adaptación que hace el estudiante de su proceso de aprendizaje al entorno que lo rodea, también cómo el docente lo hace partícipe de la acción educativa a partir de la contextualización de su realidad personal, social e institucional. Finalmente, la flexibilidad; entendida como la capacidad de dominar las habilidades o los conocimientos de acuerdo a la situación que se plantee en el aprendizaje.

Desde la posición de Páramo (s.f.) las competencias son una "una combinación de conocimientos, habilidades y actitudes" que juegan un papel fundamental para determinar el nivel de apropiación de ciertos aprendizajes en un contexto determinado, en este caso el educativo donde el estudiante usa el conocimiento de sus habilidades para convertir el aprendizaje un algo útil y experiencial. Así pues, las competencias identificaran las características particulares de cada alumno y podrán ser clasificadas como formativas desde la visión de la UNESCO (1998) que abarcan el aprender a ser y a convivir, vinculadas con valores, actitudes profesionales, sociales y filosóficas.

\section{No es tan complejo pensar en una evalua- ción de competencias desde el planeamiento didáctico}

Ante una educación que atraviesa diversos cambios y transformaciones en los procesos de enseñanza, la formación en competencias se asume con beneplácito pedagógico por los cambios que deben surgir en el currículo para todos los niveles educativos y la forma como se diseñan las estrategias para enseñar y que se produzcan los conocimientos basados en competencias. Entonces, comprendiendo claramente el concepto de evaluación y competencias ajustadas al ámbito educativo, es posible abordar ahora teóricamente la evaluación por o de competencias y describir cómo es concebida desde el planeamiento didáctico.

Concedido lo anterior, ocurrirá la discusión sobre el logro de las competencias mediante la planeación didáctica que deberá estar sujeta a un modelo pedagógico que permita adquirir las competencias correspondientes al proceso de aprendizaje. De manera que se puede pensar en la modificación de las metodologías que dirigen la enseñanza, el aprendizaje y la evaluación, lo cual implica transformar los métodos de enseñanza acordes a las habilidades, capacidades, actitudes, destrezas y valores del estudiante.

Dentro de este marco, se ha de considerar las competencias desde la posición de Beneitone, (2007) como "un saber profundo, no sólo saber qué y saber cómo, sino saber ser persona en un mundo complejo, cambiante y competitivo" donde el proceso de enseñanza-aprendizaje se convierte en el entorno donde se sustentan las competencias a desarrollar. De 
manera que surge un modelo de planeamiento didáctico para evaluar las competencias como elemento de dicho proceso, determinado por la participación activa del estudiante, el profesor, los recursos, las metodologías, el contexto, etc.; surge además, la dicotomía sobre el fin de la evaluación de competencias a partir del modelo anterior, y es establecer mediante el planeamiento didáctico si se usa con el fin de certificar el aprendizaje, o con un fin pedagógico que sería determinar el progreso de logros u objetivos alcanzados por el estudiantes.

De aquí se desprende la reflexión teórica desde una postura pedagógica sobre la evaluación de competencias que buscará constantemente la retroalimentación y la mejora del proceso de formación del estudiante. Pero antes, hay que pensar en lograr prácticas evaluativas basadas en estrategias formativas que susciten el interés metacognitivo del alumno dentro de la acción educativa. Para acercarnos a ese logro, es necesario "determinar y reflexionar en qué medidas se están cumpliendo las metas de calidad que nos hemos propuesto" plantea Ramos (s.f.), que desde el planeamiento didáctico hay que tener en cuenta el proceso de retroalimentación que ocurre dentro del aprendizaje marcado por el desempeño de las competencias puestas en acción por el estudiante para enfrentar la acción pedagógica.

Antes de pasar adelante conviene señalar a qué le llamamos planeamiento didáctico, y por qué es tan importante para la evaluación de competencias. Para su definición, comencemos por explicar que es un proceso donde se hace una interpretación de la realidad educativa, se toman decisiones en torno a las circunstancias o situaciones problemáticas y el establecimiento de oportunidades pedagógicas para dirigir el acto educativo (Díaz y
Rojas, 2002). Para el docente es evidente que se convierte en un marco de referencia global en el desarrollo de su quehacer pedagógico, pues claramente es él quien principalmente ejecuta de la enseñanza y posteriormente la evaluación de la misma y el aprendizaje.

El planeamiento didáctico lo concibe el acto educativo como el fundamento para que el docente ejerza un marco globalizado de la enseñanza, asumiendo el rol integrador de la evaluación para la planeación, ejecución y evaluación del proceso de enseñanza-aprendizaje. Procede ahora comprender la manera como se orienta la practica pedagógica desde la planeación que abarca programas y planes didácticos específicos que diseñan los docentes.

\section{Relación entre el planeamiento didáctico y la evaluación de competencias}

Ahora bien, citando a Díaz y Rojas (2002) en relación a la evaluación de competencias, el planeamiento didáctico se centra en el proceso de enseñanza aprendizaje articulando todos los saberes puestos a disposición en la práctica pedagógica. Se buscará inducir a la reflexión del aprendizaje como elemento fundamental para mejorar la calidad de esos aprendizajes. Entre tanto, las competencias deberán ser vistas como "evolución" y "cambio" donde se aplican ciertos criterios de calidad para determinar el nivel de logro del estudiante mediante el aprendizaje. Similarmente, el planeamiento didáctico es el que representa la manera como se desarrolla una verdadera practica pedagógica, con todos sus elementos teóricos, metodológicos, evaluativos y contextualizados para generar conocimientos, recolectar información valiosa, tomar decisiones y asumir una actitud crítica frente a la experiencia de aprendizaje que evalúa las competencias del estudiante, lo que 
desde el punto de vista de Deseco (2003) significaría hacer "combinación de habilidades prácticas, conocimientos, motivación, valores éticos, actitudes, emociones y otros componentes sociales y de comportamiento que se movilizan conjuntamente para logra una acción eficaz". Salvo que el fin último, será lograr la formación integral del alumno para que ponga en marcha las competencias en la vida cotidiana y profesional que le espera.

\section{CONCLUSIONES}

La evaluación de competencias desde el planeamiento didáctico deberá asumirse con criterio y rigurosidad, porque como actividad compleja, es de gran alcance por la capacidad integradora que tiene den- tro del proceso de enseñanza-aprendizaje.

El docente durante su planeación para evaluar competencias en los estudiantes debe entender cómo se involucran las necesidades, expectativas e intereses del contexto educativo con el currículo, la didáctica, la pedagogía, la evaluación, el aprendizaje y la enseñanza. Cosa parecida sucede también, que el docente para el planeamiento didáctico de una evaluación de competencias será el directamente responsable y tener la competencia para encargarse del proceso identificando el perfil del estudiante, tejiendo el mapa curricular, diseñar el plan o programa, y orientar todas las acciones hacia el logro de objetivos para el aprendizaje. Así como premeditar las competencias y educarse sobre ellas.

\section{REFERENCIAS BIBLIOGRÁFICAS}

Beneitone, P. Esquetini, C., González, J., Marty, M., Siufi, G. y Wagenaar, R. (2007). Reflexiones y perspectivas de la Educación Superior en América Latina. Bilbao: Publicaciones de la Universidad de Deusto. http://tuningacademy.org/wp-content/uploads/2014/02/TuningLAIII_Final-Report_SP.pdf

Cano, M. (2008). La evaluación por competencias en la educación superior. Profesorado, Revista de curriculum y formación del profesorado. Universitat de Barcelona. http://www.ugr.es/ recfpro/rev123COL1.pdf

Chaviano, O., Baldomir, T., Coca, O. y Gutiérrez, A. (2016). La evaluación del aprendizaje: nuevas tendencias y retos para el profesor. EDUMECENTRO, 8(4), 191-205. http://scielo.sld.cu/scielo.php?script=sci_arttext\&pid=S2077-28742016000700014\&ln$\mathrm{g}=$ es\&tlng=es.

Delors, J. (1996). La educación encierra un tesoro. Informe a la UNESCO de la Comisión Internacional sobre la Educación para el Siglo XXI. Ediciones UNESCO. https://redcampussustentable.cl/wp-content/uploads/2018/03/7-La-educaci\%C3\%B3n-encierra-un-tesoro.pdf 
La evaluación de competencias desde el planeamiento didáctico.

DeSeco (2003). La definición y selección de competencias clave. Resumen ejecutivo (OCDE) Editorial La Muralla. https://www.deseco.ch/bfs/deseco/en/index/03/02.parsys.78532. downloadList.94248.DownloadFile.tmp/2005.dscexecutivesummary.sp.pdf

Díaz, F. y Rojas G. (2002). Estrategias Docentes para un aprendizaje significativo. Mc GrawHill. https://buo.mx/assets/diaz-barriga,---estrategias-docentes-para-un-aprendizaje-significativo.pdf

Fernández, J. (2018). Evaluación del rendimiento, evaluación del aprendizaje. Ediciones AKAL. Volumen 5 de Sociedad, cultura y educación. https://books.google.com.gt/books?id=P5jaxmd7e64C

Graham, G. (1991). Condiciones para una evaluación continuada favorecedora del aprendizaje. Cuadernos de docencia universitaria. ICE y Ediciones Octaedro, S.L. https://octaedro.com/wp-content/uploads/2019/02/16513.pdf

Irigoin, M. y Vargas, F. (2000). Competencia laboral. Manual de conceptos, métodos y aplicaciones en el sector Salud. https://www.oitcinterfor.org/sites/default/files/file_publicacion/ man_ops.pdf

Martínez, E. y Cegarra-Navarro, J. (s.f.). El desarrollo de competencias transversales mediante proyectos de emprendimiento en el marco de una asignatura de dirección de operaciones. Vol 3, No 2, p. 9-13. https://www.dialnet.unirioja.es/descarga/articulo/4324836.pdf

Morales, J. (2001). La evaluación en el área de educación visual y plástica en la Educación Secundaria Obligatoria. Universidad Autónoma de Barcelona. https://www.tdx.cat/bitstream/handle/10803/5036/jjma01de16.pdf.pdf

Paramo, P. (s.f.). ¿Qué son las competencias educativas? https://www.es.scribd.com/doc/62619171/Que-son-las-Competencias-Educativas

Perrenoud, P. (2004). Diez nuevas competencias para enseñar; invitación al viaje (1a. Ed.). GRAO. https://www.uv.mx/dgdaie/files/2013/09/Philippe-Perrenoud-Diez-nuevas-competencias-para-ensenar.pdf

Ramos, C., Beresaluce, R. y Peiró, S. (s.f.). La evaluación por competencias en la Universidad. Departamento de Didáctica General y D. específicas Universidad de Alicante. https://web.ua.es/es/ice/jornadas-redes-2016/documentos/tema-3/807132.pdf

Tobón, S. (2006). Aspectos básicos de la formación basada en competencias Talca: Proyecto Mesesup, p.1-16 https://www.urosario.edu.co/CGTIC/.../aspectos_basicos_formacion_basada_competenci as.p 
La evaluación de competencias desde el planeamiento didáctico.

Caicedo J.

Conceptualización e importancia

Tyler, R. (1950). Principio básico del plan de estudios y de la enseñanza. Universidad de Chicago. http://blogs.ubc.ca/ewayne/files/2009/02/tyler_001.pdf

UNESCO (1998). Declaración mundial sobre la educación superior en el siglo XXI. https://www.usfq.edu.ec/sites/default/files/2020-06/pea_013_0015.pdf 